\title{
LONG-SLIT SPECTROSCOPY OF IC 5135 AND NGC 4388
}

\author{
JOSEPH C. SHIELDS and ALEXEI V. FILIPPENKO \\ Department of Astronomy, University of California \\ Berkeley, CA 94720 U.S.A.
}

The nucleus of the peculiar Sa galaxy IC 5135 has strong emission lines whose relative intensities resemble those of Seyfert 2 galaxies (Phillips, Charles, and Baldwin 1983). However, the galaxy exhibits relative line intensities such as [O III] $\lambda 5007 / \mathrm{H} \beta=6.5$ and $\mathrm{He}$ iI $\lambda 4686 / \mathrm{H} \beta=0.17$ that are considerably smaller than typical Seyfert 2 values of 11 and 0.3 , respectively (Koski 1978). New, high-resolution spectra (FWHM $\approx 2 \AA$ ) obtained at Las Campanas Observatory reveal that the $\mathrm{H} \beta$ emission-line profile actually consists of a relatively strong, narrow component $\left(F W H M \approx 250 \mathrm{~km} \mathrm{~s}^{-1}\right.$ ) superposed on a much weaker, broad base (FWHM $\approx 1000 \mathrm{~km} \mathrm{~s}^{-1}$; Fig. 1). A similar two-component profile is evident in [O I] $\lambda 6300$, whose narrow core probably comes from circumnuclear $\mathrm{H}$ II regions that produce little [O III] and He II emission. The strong, high-order Balmer absorption lines are consistent with the presence of a young stellar population in the nucleus.

Deconvolution of the $\mathrm{H} \alpha+[\mathrm{N}$ II $] \lambda \lambda 6548,6583$ blend reveals additional kinematic differences between gas emitting different lines and line components. Attempts to model the blend with scaled profiles of $\mathrm{H} \beta$ (to match $\mathrm{H} \alpha$ ) and [O III] $\lambda 5007$ (to match [N II]) failed, primarily because the [O III] lines are much more symmetric than the [N II] lines. Use of $\mathrm{H} \beta$ alone to match the $\mathrm{H} \alpha$ and [N II] profiles also did not work well; the observed $\mathrm{H} \alpha$ $+[\mathrm{N} \mathrm{II}]$ blend has too much broad emission. A good fit to the observed profile, however, was produced by deconvolving the [S II] $\lambda \lambda 6716,6731$ blend with an iterative technique, and using the resulting synthetic [S II] profile to model the [N II] components. The derived [S II] profile shows a narrow core, and an asymmetric broad base reminiscent of the $\mathrm{H} \beta$ profile. It is likely that the narrow core is produced largely by $\mathrm{H}$ II regions around $\mathrm{O}$ and B stars, whereas the broad base is associated with the Seyfert nucleus.

The interpretation of IC 5135 as a composite of Seyfert and starburst phenomena is further supported by spatially resolved spectra obtained at Cerro Tololo. As the distance from the nucleus increases, [O III] $\lambda 5007 / \mathrm{H} \beta$ and [N II] $\lambda 6583 / \mathrm{H} \alpha$ decrease to values consistent with metal-rich H II regions. On the other hand, the presence of

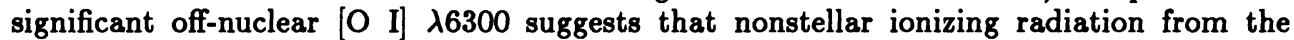
nucleus also influences the gas. The observed increase of [O I] $\lambda 6300 /[O$ III $] \lambda 5007$ and [O II] $\lambda 3727 /[\mathrm{O}$ III] $\lambda 5007$ with distance from the nucleus is a consequence of the radial dilution of the nonstellar radiation; the ionization parameter decreases with increasing distance from the nucleus. It is possible that the coexistence of a strong $H$ II region component with an active nucleus in IC $\mathbf{5 1 3 5}$ is simply a random superposition of unrelated phenomena. However, this galaxy represents an interesting case for more detailed study in light of recent suggestions that nuclear starbursts may evolve into, and feed, an active nucleus (e.g., Weedman 1983, Scoville and Norman 1988).

NGC 4388 is a highly-inclined disk galaxy $\left(i \approx 72^{\circ}\right.$, major axis PA $\left.\approx 92^{\circ}\right)$ with probable membership in the Virgo cluster. Previous work demonstrates that its nucleus shows strong forbidden lines typical of Seyfert 2 galaxies. A weak component of broad $\mathrm{H} \alpha$ with full-width near zero intensity $(F W Z I) \approx 6000 \mathrm{~km} \mathrm{~s}^{-1}$, typical of Seyfert 1 galaxies, 480 
was found in the apparent nucleus of NGC 4388 by Filippenko and Sargent (1985). New long-slit spectra acquired at multiple locations in the central region of NGC 4388 show that additional broad $\mathrm{H} \alpha$ emission (FWZI $\approx 3000-5000 \mathrm{~km} \mathrm{~s}^{-1}$; Fig. 2) is visible out to roughly $6^{\prime \prime}$ from the apparent nucleus in $\mathrm{PA} \approx 0^{\circ}-70^{\circ}$ and at other locations northwest, southeast, and directly south of the apparent nucleus.

Extended broad-line emission is remarkable since the broad-line regions of all but one known Seyfert 1 galaxies are unresolved. The exception is Mkn 110, whose off-nuclear broad $\mathrm{H} \beta$ line is attributed by Hutchings and Craven (1988) to mass motions along a jet. We interpret the observed off-nuclear broad H $\alpha$ in NGC 4388 as radiation from an obscured Seyfert 1 nucleus that is scattered into our line of sight, most likely by dust. The true Seyfert nucleus is probably displaced from the apparent optical nucleus, judging from an offset of the apparent nucleus from the galaxy's center of symmetry, and an offset in the same sense of the apparent nucleus from the 6-cm radio core. The true nucleus is probably obscured by the normal ISM of the galaxy's disk. A scattering origin of the extended broad H $\alpha$ may be tested with spectropolarimetric measurements.

NGC 4388 strongly resembles NGC 1068 with its nucleus dominated by Seyfert 2 spectral features, radio plumes, cones of high-ionization gas extending from the nucleus, obscured broad-line region, and off-nuclear scattering regions. To explain broad-line region obscuration in NGC 1068, Antonucci and Miller (1985) posited a thick torus surrounding the nucleus of this relatively low-inclination system. In NGC 4388, however, obscuration of the nucleus is readily explained by normal disk material. NGC 4388 thus does not provide a strong test of the proposition that all Seyfert 2 galaxies contain obscured broad-line regions.

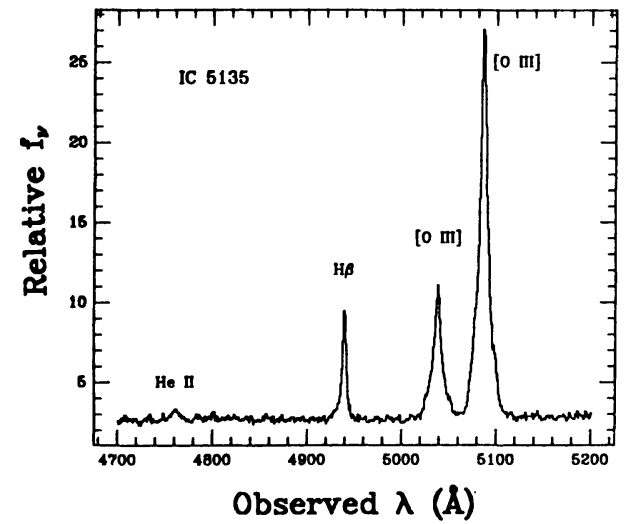

Fig. 1- Spectrum of the nucleus of IC 5135, showing emission lines with varying proportions of broad and narrow velocity components.

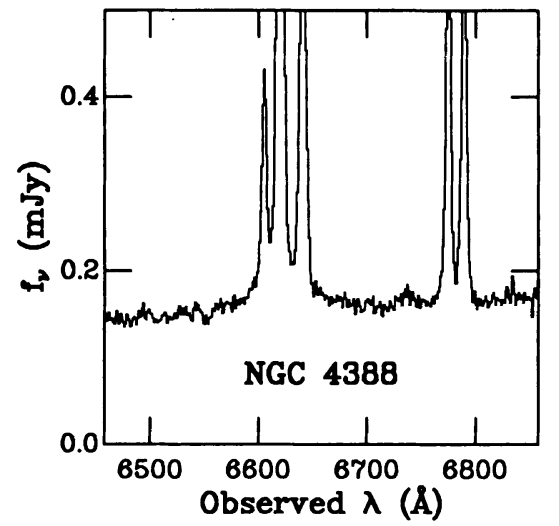

Fig. 2- Spectrum averaged over 6.4 sq. arcsec centered $3^{\prime \prime} \mathrm{N}, 4^{\prime \prime} \mathrm{E}$ of the apparent nucleus of NGC 4388, showing broad wings on the [N II] $+\mathrm{H} \alpha$ blend.

\section{REFERENCES}

Antonucci, R. R. J., and Miller, J. S. 1985, Ap. J., 297, 621.

Filippenko, A. V., and Sargent, W. L. W. 1985, Ap. J. Suppl., 57, 503.

Hutchings, J. B., and Craven, S. E. 1988, A. J., 95, 677.

Koski, A. T. 1978, Ap. J., 223, 56.

Phillips, M. M., Charles, P. A., and Baldwin, J. A. 1983, Ap. J., 266, 485.

Scoville, N., and Norman, C. 1988, Ap. J., 332, in press.

Weedman, D. W. 1983, Ap. J., 266, 479. 